\title{
El Cinismo del Empleado y su Relación con Factores Demográficos
}

\section{Employee Cynicism and its Relationship with Demographic} Factors

\author{
Y. Hernández-Pantoja', M. M. Vargas-Salgado', K. G. Gómez-Bull ${ }^{1}$ \\ ${ }^{1}$ Universidad Autónoma de Ciudad Juárez
}

\section{RESUMEN}

Este trabajo de investigación tuvo como objetivo analizar si el cinismo organizacional es diferente de acuerdo con el sexo, el año de nacimiento, la antigüedad y la escolaridad de los empleados operativos que laboran en una empresa de manufactura de Ciudad Juárez. El estudio fue de tipo cuantitativo, no experimental, transversal y descriptivo. Se aplicó un instrumento de 6 ítems que valoró el cinismo del empleado a una muestra no probabilística por conveniencia de 102 empleados operativos que laboran en la industria manufacturera. Se realizó análisis de varianza (ANOVA), considerándose como variables el sexo, el año de nacimiento, la antigüedad y la escolaridad de los sujetos de estudio. Los resultados revelan que no existen diferencias significativas de acuerdo con sexo, el año de nacimiento y la antigüedad, y por otra parte se encontraron diferencias significativas con la variable de escolaridad. En conclusión, se encontró que entre menos escolaridad posean los empleados, estos son más cínicos que aquellos que cuentan con estudios superiores.

PALABRAS CLAVE: Cinismo; sexo; año de nacimiento; antigüedad; escolaridad.

\begin{abstract}
This research work aimed to analyze whether organizational cynicism is different according to the sex, year of birth, seniority and schooling of operational employees working in a manufacturing company in Ciudad Juarez. The study was quantitative, non-experimental, transversal and descriptive, and a 6-item instrument was applied that assessed employee cynicism to a non-probabilistic sample for the convenience of 102 operational employees, who work in the manufacturing industry. Analysis of variance [ANOVA] was carried out considering as variables the sex, year of birth, seniority and schooling of the study subjects. The results reveal that there are no significant differences according to sex, year of birth and seniority. Otherwise, significant differences were found with the schooling variable. In conclusion, it was found that the less schooling employees have, these are more cynical, than those with higher education.
\end{abstract}

KEYWORDS: Cynicism; sex; year of birth; antiquity; scholarship.

Correspondencia:

DESTINATARIO: María Marisela Vargas Salgado INSTITUCIÓN: Universidad Autónoma de Ciudad Juárez DIRECCIÓN: Avenida Universidad y Avenida Heroico Colegio Militar s/n, Zona Chamizal, C. P. 32300, Ciudad Juárez, Chihuahua, México.

CORREO ELECTRÓNICO: maria.vargas@uacj.mx
Fecha de recepción:

26 de julio de 2019

Fecha de aceptación:

3 de septiembre de 2019 


\section{INTRODUCCIÓN}

Hoy en día las organizaciones se enfrentan constantemente a cambios desafiantes para alcanzar sus objetivos y permanecer en un contexto competitivo, en el cual en ocasiones las empresas se ven en la necesidad de disminuir el personal como una alternativa viable para su entorno. Sin embargo, de un tiempo a la fecha el mundo organizacional globalizado ha impactado en los trabajadores, provocando en estos una reducción en la confianza para con el empleador, aumentando el recelo, la suspicacia y la decepción ${ }^{[1]}$. Además, durante los últimos años se han presentado cambios significativos en la tecnología, la información, la comunicación y la sociedad, en particular en las cuestiones demográficas, impactando considerablemente en el mundo laboral ${ }^{[2,3]}$.

Maslach ${ }^{[4]}$ y Aguilar, Rodríguez y Salanova ${ }^{[5]}$ refieren que el cinismo representa una actitud negativa, progresiva o excesivamente apática a diversos aspectos del trabajo, como tareas y objetivos organizacionales. Este comportamiento se puede desarrollar cuando hay un exceso de preocupación o bien cuando el empleado realiza diversas actividades a la vez. Por otra parte, se piensa que el trabajador tiene la creencia que ha fallado con los principios de honestidad y justicia, por lo que le nace el desinterés por realizar sus actividades, reaccionando a la defensiva a causa de una sobrecarga de trabajo, largas horas de actividades, recompensas no cumplidas y falta de liderazgo ${ }^{[1,6]}$.

En la literatura es posible identificar tres facetas del cinismo: creencia, afecto y comportamiento. Con respecto a la primera, hace referencia a la opinión de que la empresa carece de integridad, que es injusta y que esta es utilizada a conveniencia de la firma. La segunda está representada por emociones negativas: las personas cínicas experimentan angustia, disgusto e incluso vergüenza cuando piensan en la empresa. Finalmente, en la tercera, los empleados pueden utilizar el humor, especialmente de forma sarcástica, para expresar sus actitudes cínicas, es decir, constantemente pueden hacer comentarios pesimistas sobre el futuro de la organización ${ }^{[7]}$.

Las personas cínicas se pueden caracterizar por sentir frustración, desilusión, desprecio y desconfianza e incluso hablan mal de la organización una vez que se sienten traicionadas, por lo que tienen la sensación de que su trabajo no tiene valor alguno ${ }^{[8]}$. Además los empleados buscan la forma de reducir la cantidad de tiempo que pasan en el entorno laboral y por ende su desempeño será bajo ${ }^{[4,9]}$.

Chiaburu, Peng, Oh, Banks y Lomeli ${ }^{[10]}$ refieren que últimamente en el entorno laboral se han incrementado cada vez más las actitudes cínicas, especialmente en áreas de trabajo donde prevalece la desconfianza, escándalos y comportamientos oportunistas y que como consecuencia baja el rendimiento, la satisfacción en el trabajo y el compromiso organizacional de los empleados para con la firma. No obstante, Brandes y Das [11] indican que el cinismo puede mejorar el rendimiento, especialmente cuando hay una necesidad de desafiar y cambiar procedimientos de forma radical. Sin embargo, los trabajadores que presentan un alto nivel de cinismo tienden a ausentarse, abandonar la organización, cambiar de trabajo e incluso dejar temprano sus actividades, es decir, no existe disposición alguna. Además, es de resaltar que hay características, como la edad, que muestran que los empleados más jóvenes tienden a ser más cínicos al principio de la carrera laboral y con respecto al sexo se considera que los hombres son más cínicos que las mujeres ${ }^{[4]}$. Algunos individuos cínicos piensan constantemente que existen motivos ocultos para las acciones y se caracterizan por cuestionar las decisiones que toma la alta gerencia ${ }^{[7]}$.

De acuerdo con lo anterior, este trabajo de investigación busca analizar si el cinismo organizacional es diferente de acuerdo con el sexo, el año de nacimiento, la antigüedad y la escolaridad de los empleados operativos que laboran en una empresa de manufactura de Ciudad Juárez.

\section{METODOLOGÍA}

El diseño de esta investigación es de tipo cuantitativo, no experimental, transversal y descriptivo. La muestra fue no probabilística y se realizó un muestreo por conveniencia a trabajadores que laboran en una empresa de manufactura de Ciudad Juárez, considerando como sujetos de estudio a 102 empleados de nivel operativo. Como técnica de recolección de datos se utilizó una encuesta, la cual se aplicó directamente a cada uno de los participantes en su entorno laboral.

El instrumento integró 6 ítems (Tabla 1) con un formato tipo Likert de cinco puntos con opciones a res- 
ponder: nunca, muy pocas veces, algunas veces, casi siempre y siempre. El cinismo del empleado se midió a través de la escala propuesta por Maslach y Jackson ${ }^{[9]}$. Hernández, Fernández y Baptista ${ }^{[12]}$ señalan que cada instrumento de medición deberá de exhibir un porcentaje de fiabilidad aceptable que en este caso el coeficiente de Cronbach presenta un 0.750, lo cual indica que el instrumento se considera fiable. Además, se integraron a este algunos factores demográficos tales como sexo, año de nacimiento y antigüedad. Asimismo, se utilizó la herramienta estadística SPSS para el análisis e interpretación de los datos tanto descriptivos como inferenciales referente a los resultados recabados.

TABLA 1.

Ítems de la Escala de Cinismo del Empleado

\begin{tabular}{|l|l|}
\hline CE1 & $\begin{array}{l}\text { Desde que comencé este trabajo he ido perdiendo el } \\
\text { interés en él. }\end{array}$ \\
\hline CE2 & He ido perdiendo el entusiasmo. \\
\hline CE3 & Solo quiero hacer mis actividades y que no me molesten. \\
\hline CE4 & $\begin{array}{l}\text { Me burlo de mí mismo pensando que mi trabajo sirve } \\
\text { para algo. }\end{array}$ \\
\hline CE5 & Dudo sobre el valor de lo que realizo. \\
\hline CE6 & Siento que mis actividades no sirven para nada \\
\hline
\end{tabular}

Fuente: Elaboración propia a partir de Maslach y Jackson ${ }^{[9]}$.

En este trabajo de investigación se proponen las siguientes hipótesis:

H0 a: El cinismo organizacional no es distinto en relación con el sexo.

$\mathrm{H} 0$ b: El cinismo organizacional no es distinto en función del año de nacimiento.

$\mathrm{H} 0 \mathrm{c}$ : El cinismo organizacional no es distinto en relación con la antigüedad.

Ho d: El cinismo organizacional no es distinto en función de la escolaridad.

Para probar dichas diferencias, se utilizó el análisis de varianza de un factor, herramienta que permite identificar si existen o no diferencias significativas en los distintos grupos.

\section{RESULTADOS Y DISCUSIÓN}

En la Tabla 2 se presenta las características demográficas de los sujetos de estudio, donde el $63.7 \%$ corresponde a hombres, mientras que el $50 \%$ concierne a las personas nacidas entre 1960 y 1980 , es decir, que pertenecen a la denominada generación X, el 52\% de los su- jetos manifesta tener entre 1 y 5 años de antigüedad en la organización, mientras que el $52.9 \%$ son empleados que cuentan con estudios menor a bachillerato.

TABLA 2.

Estadísticos Descriptivos de Cinismo del Empleado $n=102$

\begin{tabular}{|c|c|c|}
\hline Característica & $\begin{array}{c}\text { No. DE } \\
\text { PERSONAS }\end{array}$ & $\%$ \\
\hline \multicolumn{3}{|l|}{ Sexo } \\
\hline Femenino & 37 & 36.3 \\
\hline Masculino & 65 & 63.7 \\
\hline \multicolumn{3}{|l|}{ Año de nacimiento } \\
\hline Antes de 1960 & 5 & 4.9 \\
\hline Entre 1960 y 1980 & 51 & 50.0 \\
\hline Entre 1981 y 1999 & 44 & 43.1 \\
\hline Después del año 2000 & 2 & 2.0 \\
\hline \multicolumn{3}{|l|}{ Antigüedad } \\
\hline Menor a 1 año & 14 & 13.7 \\
\hline De 1 año a 5 años & 53 & 52.0 \\
\hline De 6 a 10 años & 22 & 21.6 \\
\hline De 11 a 15 años & 11 & 10.8 \\
\hline De 16 a 20 años & 0 & 0.0 \\
\hline De 20 años en adelante & 2 & 2.0 \\
\hline \multicolumn{3}{|l|}{ Escolaridad } \\
\hline Menor a bachillerato & 54 & 52.9 \\
\hline Bachillerato & 30 & 29.4 \\
\hline Carrera técnica & 6 & 5.9 \\
\hline Carrera profesional no terminada & 6 & 5.9 \\
\hline Otros & 6 & 5.9 \\
\hline
\end{tabular}

Fuente: Elaboración propia.

En la Tabla 3 se muestran los descriptivos de la variable cinismo del empleado, en donde se observa que el valor mínimo fue de 1 en todos los casos, mientras que el valor máximo fue de 5 , a excepción de los ítems 1 y 5 . Respecto a la media, la más alta fue del 2.34 en el ítem 3: "Solo quiero hacer mis actividades y que no me molesten", por lo que los sujetos indicaron que casi nunca los interrumpen al momento de realizar las actividades, dado que estos desean estar concentrados en las tareas y no ser molestados. El ítem con la media más baja es el 6: "En mi trabajo siento que mis actividades no sirven para nada", con un valor del 1.39 , lo cual indica que los trabajadores perciben que las tareas que realizan no tienen sentido y mucho menos son importantes. 
TABLA 3.

Estadísticos Descriptivos de Cinismo del Empleado $n=102$

\begin{tabular}{|l|l|l|l|l|}
\hline \multicolumn{1}{|c|}{ Ítems } & Mínimo & Máximo & Media & $\begin{array}{l}\text { Desviación } \\
\text { ESTÁndAR }\end{array}$ \\
\hline $\begin{array}{l}\text { 1. Desde que comencé mi } \\
\text { trabajo he ido perdiendo } \\
\text { el interés en él. }\end{array}$ & 1 & 4 & 1.70 & .920 \\
\hline $\begin{array}{l}\text { 2. En mi trabajo he ido } \\
\text { perdiendo el entusiasmo. }\end{array}$ & 1 & 5 & 1.83 & .924 \\
\hline $\begin{array}{l}\text { 3. Solo quiero hacer mis } \\
\text { actividades, que no me } \\
\text { molesten. }\end{array}$ & 1 & 5 & 2.34 & 1.411 \\
\hline $\begin{array}{l}\text { 4. Me burlo de mí mismo } \\
\text { pensando que mi trabajo } \\
\text { sirve para algo. }\end{array}$ & 1 & 5 & 1.53 & .972 \\
\hline $\begin{array}{l}\text { 5. En mi trabajo dudo } \\
\text { sobre el valor de lo que } \\
\text { realizo. }\end{array}$ & 1 & 4 & 1.47 & .805 \\
\hline $\begin{array}{l}\text { 6. En mi trabajo siento } \\
\text { que mis actividades no } \\
\text { sirven para nada. }\end{array}$ & 1 & 5 & 1.39 & .834 \\
\hline
\end{tabular}

Fuente: Elaboración propia.

Para valorar las diferencias significativas a nivel constructo, se construyó un índice de cinismo del empleado. Se procedió a realizar un Análisis Factorial Exploratorio (AFE) y los datos revelaron que el KMO fue de 0.718 , es decir, el índice es superior al punto de corte, en tanto que el Test de Esfericidad de Barlett tuvo una $p=0.000$ con una Chi-cuadrada de 215.913 con 15 grados de libertad (g.l.). Posteriormente se procedió a realizar el análisis de varianza de un factor. Los resultados revelan que no existen diferencias estadísticamente significativas en el cinismo, con base en las variables de sexo, año de nacimiento y antigüedad. Por el contrario, se encontraron diferencias significativas entre los grupos a partir de la escolaridad, la cual se clasifica en cinco grupos: 1-menor a bachillerato, 2-bachillerato, 3-carrera técnica, 4-carrera profesional terminada y 5-otros. En la Tabla 4 se muestran los resultados del análisis de varianza, con un nivel $p=0.010$.

TABLA 4.

Prueba de ANOVA Entre los Grupos Respecto al Cinismo CON BASE EN LA ESCOLARIDAD

\begin{tabular}{|l|l|l|l|l|l|}
\hline & $\begin{array}{c}\text { SUMA DE } \\
\text { CUADRADOS }\end{array}$ & G.L. & $\begin{array}{c}\text { MEDIA } \\
\text { CUADRÁtICA }\end{array}$ & F & Sig. \\
\hline $\begin{array}{l}\text { Entre } \\
\text { grupos }\end{array}$ & 12.871 & 4 & 3.218 & 3.542 & .010 \\
\hline $\begin{array}{l}\text { Dentro de } \\
\text { los grupos }\end{array}$ & 88.129 & 97 & .909 & & \\
\hline Total & 101.000 & 101 & & & \\
\hline
\end{tabular}

$\mathrm{F}=$ prueba de Fischer. Sig. = significancia. Fuente: Elaboración propia.
Como se muestra en la Tabla 5, de acuerdo con la prueba Tukey existen diferencias estadísticamente significativas entre los grupos 1 y 2 , no así en los 3,4 y 5 , es decir, los trabajadores que tienen menos estudios académicos son más cínicos que los empleados que están más preparados profesionalmente.

TABLA 5.

Comparaciones Múltiples de Acuerdo con la Prueba Tukey Del Cinismo Basadas en la Escolaridad

\begin{tabular}{|c|c|c|c|c|c|c|}
\hline (I) & (J) & \multirow{2}{*}{$\begin{array}{c}\text { DIFERENCIAS } \\
\text { DE MEDIAS } \\
\text { (I-J) }\end{array}$} & \multirow{2}{*}{$\begin{array}{c}\text { ERROR } \\
\text { ESTÁNDAR }\end{array}$} & \multirow{2}{*}{ SIG. } & \multicolumn{2}{|c|}{$\begin{array}{c}95 \% \text { INTERVALO DE } \\
\text { CONFIANZA }\end{array}$} \\
\hline \multicolumn{2}{|c|}{ ESCOLARIDAD } & & & & $\begin{array}{c}\text { LÍMITE } \\
\text { INFERIOR }\end{array}$ & \begin{tabular}{|l} 
LÍMITE \\
SUPERIOR
\end{tabular} \\
\hline \multirow[t]{4}{*}{1} & 2 & $-.73827501^{\star}$ & .21704833 & .008 & -1.3416165 & -.1349335 \\
\hline & 3 & .28987868 & .41018279 & .955 & -.8503295 & 1.4300869 \\
\hline & 4 & .01187403 & .41018279 & 1.000 & -1.1283342 & 1.1520822 \\
\hline & 5 & -.45091775 & .41018279 & .806 & -1.5911260 & 6892905 \\
\hline \multirow[t]{4}{*}{2} & 1 & $.73827501^{\star}$ & .21704833 & .008 & .1349335 & 1.3416165 \\
\hline & 3 & 1.02815369 & \begin{tabular}{|l}
.42627446 \\
\end{tabular} & .121 & -.1567854 & 2.2130928 \\
\hline & 4 & .75014904 & \begin{tabular}{|l}
.42627446 \\
\end{tabular} & .403 & -.4347901 & 1.9350882 \\
\hline & 5 & .28735726 & .42627446 & .962 & -.8975819 & \begin{tabular}{|l|}
1.4722964 \\
\end{tabular} \\
\hline \multirow[t]{4}{*}{3} & 1 & -.28987868 & .41018279 & .955 & -1.4300869 & .8503295 \\
\hline & 2 & -1.0281536 & .42627446 & .121 & -2.2130928 & .1567854 \\
\hline & 4 & -.27800465 & 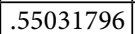 & .987 & -1.8077545 & 1.2517452 \\
\hline & 5 & -.74079643 & \begin{tabular}{|l|}
.55031796 \\
\end{tabular} & .663 & -2.2705463 & .7889534 \\
\hline \multirow[t]{4}{*}{4} & 1 & -.01187403 & .41018279 & 1.000 & -1.1520822 & 1.1283342 \\
\hline & 2 & -.75014904 & .42627446 & .403 & -1.9350882 & .4347901 \\
\hline & 3 & .27800465 & .55031796 & .987 & -1.2517452 & 1.8077545 \\
\hline & 5 & -.46279178 & .55031796 & .917 & -1.9925416 & 1.0669581 \\
\hline \multirow[t]{4}{*}{5} & 1 & .45091775 & .41018279 & .806 & -.6892905 & 1.5911260 \\
\hline & 2 & -.28735726 & .42627446 & .962 & -1.4722964 & .8975819 \\
\hline & 3 & .74079643 & .55031796 & .663 & -.7889534 & 2.2705463 \\
\hline & 4 & .46279178 & .55031796 & .917 & -1.0669581 & 1.9925416 \\
\hline
\end{tabular}

*La diferencia de medias es significativa a un nivel 0.05. Fuente: Elaboración propia.

\section{CONCLUSIONES}

Con el paso del tiempo, el cinismo se ha incremento de manera significativa en las empresas, pues esta variable es considerada como una actitud negativa que conduce a resultados no deseados, a una menor lealtad y bajo rendimiento, por lo que atribuye a la desconfianza, escándalos y comportamientos oportunistas ${ }^{[13]}$.

En este trabajo de investigación se tuvo como objetivo analizar si el cinismo organizacional es diferente de acuerdo con el sexo, año de nacimiento, antigüedad y escolaridad de los empleados operativos que laboran en una empresa de manufactura ubicada en Ciudad Juárez. Los resultados indican que no existen diferencias significativas estadísticamente de acuerdo con las variables sexo, año de nacimiento y antigüedad. No obstante, se encontró que el cinismo de los empleados es estadísticamente significativo con la escolaridad de los sujetos, especialmente en aquellos que tienen me- 
nos estudios, es decir, los trabajadores que cuentan con una preparación académica inferior a bachillerato son más cínicos que aquellos que cuenten con grados académicos superiores. Por tanto, se rechaza la hipótesis $\mathrm{H} 0 \mathrm{~d}$ : El cinismo organizacional no es distinto en función de la escolaridad.

Queda patente que en los últimos años se ha destacado que la conducta del cinismo puede darse a manera de defensa de los empleados y que dicha actitud de pesimismo y desesperanza hacia cambios organizacionales puede afectar de manera negativa y ocasionar, en consecuencia, bajo rendimiento en el área laboral. Esta reacción de defensa puede deberse a los pocos conocimientos y preparación con la que cuentan los trabajadores para actuar ante diversas eventualidades que se presentan en el entorno laboral y, por ello, les resulta mejor actuar de forma cínica.

En general, se puede decir que los empleados menos preparados profesionalmente son mas cínicos que aquellos trabajadores que lo están. Para futuras investigaciones se recomienda integrar muestras probabilísticas y contextos diferentes y además sería conveniente integrar la variable de posición laboral y vincular el cinismo con otros constructos como injusticia y satisfacción laboral, entre otros.

\section{REFERENCIAS}

[1] S. Salessi y A. Omar, "Cinismo organizacional: consecuencias sobre la salud mental de los trabajadores", Psicología y Salud, vol. 24, pp. 269-277, 2014.

[2] P. R. Gil-Monte, "Riesgos psicosociales en el trabajo y salud ocupacional", Revista Peruana de Medicina Experimental y Salud Pública, vol. 29, pp. 237-241, 2012.

[3] J. Hartley, D. Jacobson, B. Klandermans y T. Van Vuuren, Job insecurity: Coping with jobs at risk. USA: Sage Publications Ltd, 1990.

[4] C. Maslach, "Comprendiendo el burnout", Ciencia \& Trabajo, vol. 11, pp. 37-43, 2009.
[5] A. Aguilar, A. M. Rodríguez, y M. Salanova, "Estilos de liderazgo y riesgos psicosociales en los empleados", Jornades de Foment de la Investigació, Universitat Jaume I, 2002. [En línea]. Disponible en: http://www.integraorg.com/wp-content/docs/estilos de liderazgo y riesgos psicosociales.pdf

[6] S. Cartwright y N. Holmes, "The meaning of work: The challenge of regaining employee engagement and reducing cynicism", Human Resource Management Review, vol. 16, pp. 199-208, 2006.

[7] J. Dean, P. Brandes, y R. Dharwadkar, "Organizational Cynicism", The Academy of Management Review, vol. 23, pp. 341-352, 1998.

[8] L. M. Andersson, "Employee cynicism: An examination using a contract violation framework", Human relations, vol. 49, pp. 1395-1418, 1996.

[9] C. Maslach y S. E. Jackson, "The measurement of experienced burnout", Journal of organizational behavior, vol. 2, pp. 99-113, 1981.

[10] D. S. Chiaburu, A. C. Peng, I.-S. Oh, G. C. Banks y L. C. Lomeli, "Antecedents and consequences of employee organizational cynicism: A meta-analysis", Journal of Vocational Behavior, vol. 83, pp. 181-197, 2013.

[11] P. Brandes y D. Das, "Locating behavioral cynicism at work: Construct issues and performance implications", in Employee health, coping and methodologies, P. Perrewé y D. Ganster, eds. Bingley: Emerald Group Publishing Limited, 2006, pp. 233-266. https://doi. org/10.1016/S1479-3555(05)05007-9

[12] R. Hernández, C. Fernández, y P. Baptista, Metodología de la investigación, 5. ${ }^{\mathrm{a}}$ ed. México: Mc Graw Hill, 2010.

[13] A. Acaray y S. Yildirim, "The impact of personality traits on organizational cynicism in the education sector", World Journal of Entrepreneurship, Management and Sustainable Development, vol. 13, pp. 65-76, 2017. 